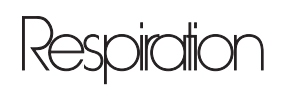

\title{
Open Letter to the Members of the GOLD Committee
}

\section{Dear Colleagues, Members of the GOLD Committee}

This letter arises from discussions and correspondence between colleagues involved in respiratory research or the diagnosis and treatment of lung diseases, as well as from a review of the literature on COPD. As discussed below, it is written in the hope that we can persuade members of the GOLD committee to vote to change the method by which mild airway obstruction is defined by the GOLD guidelines.

We very much welcome the continued efforts of the GOLD group to stimulate interest and awareness of the high prevalence of COPD, its morbidity, effects on quality of life and on mortality. There is no doubt that COPD is a major public health problem of which the public, health workers and health authorities are insufficiently aware. It is therefore an important achievement that WHO, ERS, ATS, APSR, ALAT, WONCA and many distinguished individuals have joined forces to increase awareness about the burden of disease by publishing reports and guidelines for diagnostic procedures and interventions which have been adopted by numerous international and national organisations.

However, there is one area which has given rise to continuous published criticism: the criterion for confirming airway obstruction. It is well known that the $\mathrm{FEV}_{1} / \mathrm{FVC}$ ratio declines with increasing age and height, even in healthy lifelong non-smokers, in whom the lower limit of normal drops below a ratio of 0.7 from about 45 years of age [1-6]. It has been shown [4-27] that using the fixed ratio causes up to $50 \%$ over-diagnosis (misclassification) above that age. Adult smokers suspected of having COPD are not at increased risk of respiratory symptoms, respiratory morbidity, or all-cause mortality until the ratio falls below the age-corrected fifth percentile lower limit of normal [26, 28].

The present GOLD guidelines on the spirometric assessment of airway obstruction are scientifically untenable [1, 29-31] and have given rise to editorials in Chest [32], the European Respiratory Journal [17], the American Journal of Respiratory and Critical Care Medicine [33], COPD: Journal of Chronic Obstructive Pulmonary Disease [34], and Respiratory Care [35] with a plea for revision. The very significant over-diagnosis in elderly subjects due to this guideline is akin to selling sickness. There is considerable psychological impact, and there are wider health consequences of incorrectly being labeled as having COPD, a syndrome associated with a poor prognosis with regard to morbidity, quality of life and mortality and therefore a psychological burden for the subjects, their family and wider environment. Subjects erroneously labeled become a target for individual and lifelong interventions which are associated with side effects. This is all the more unacceptable since evidence for the long-term effectiveness of treatment of mild COPD, apart from smoking cessation, is lacking $[28,36]$. Erroneous interventions also constitute an unnecessary financial burden on society.

\section{KARGER}

Fax +41613061234

E-Mail karger@karger.ch

www.karger.com
(C) 2010 S. Karger AG, Base

0025-7931/10/0804-0265\$26.00/0

Accessible online at:

www.karger.com/res
Philip H. Quanjer

Kervel 19

NL-7443 GT Nijverdal (The Netherlands)

E-Mail pquanjer@xs4all.nl 
We applaud the GOLD committee for raising interest in COPD research. However, over-diagnosis will lead to the inclusion of subjects who do not have COPD into the research pool, thereby adding noise to any signals that researchers are looking for when trying to unravel the causes of COPD and hence find potential treatments. Also problematic is excluding younger subjects who may have airway obstruction (false negatives) when the fixed ratio is used $[4-5,10,16,18,21,37-40]$. For research purposes, it is far better to limit recruitment to subjects who definitely have the disease, but this urgently requires adjustment of the present guideline on a fixed $\mathrm{FEV}_{1} / \mathrm{FVC}$ ratio.

We appreciate the consequences of changing course when so many societies and organizations will be affected by replacing the fixed ratio by the lower limit of normal, and, more importantly, general practitioners and clinicians may have to review and revise previous diagnoses. However, in the light of new evidence it is never too late to change a decision made in good faith. We are therefore appealing to you, members of the GOLD committee, to change the method by which mild airway obstruction is defined by the GOLD guidelines in order to abandon the fixed ratio forever in favor of the lower limit of normal.

\section{Sincerely yours,}

\section{The Pulmonaria Group}

Philip H. Quanjer, MD, PhD, Department of Pulmonary Diseases and Department of Paediatrics, Erasmus Medical Centre, Erasmus University, Rotterdam, The Netherlands

Paul L. Enright, MD, PhD, College of Public Health, The University of Arizona, Tucson, AZ, USA

Martin R. Miller, MD, PhD, Department of Medicine, University Hospital Birmingham NHS Trust, Birmingham, UK

Janet Stocks, PhD, Portex Respiratory Unit, UCL, Institute of Child Health, London, UK

Gregg Ruppel, RRT, RPFT, Pulmonary Function Laboratory, Saint Louis University Hospital, St. Louis, MO, USA

Maureen P. Swanney, PhD, Respiratory Physiology Laboratory, Christchurch Hospital, Canterbury District Health Board, Christchurch, New Zealand

Robert O. Crapo, Pulmonary Division, LDS Hospital and University of Utah, Salt Lake City, UT, USA

Ole F. Pedersen, MD, DMSc, Institute of Public Health, Department of Environmental and Occupational Medicine, University of Aarhus, Aarhus, Denmark

Emanuela Falaschetti, MSc, Department of Epidemiology and Public Health, University College London, London, UK

Jan P. Schouten, PhD, Department of Epidemiology, University Medical Center Groningen, University of Groningen, Groningen, The Netherlands

Robert L. Jensen, MD, PhD, Pulmonary Division, LDS Hospital and University of Utah, Salt Lake City, UT, USA

\section{Organizations}

ANZSRS: Australian and New Zealand Society of Respiratory Science

ARTP: Association for Respiratory Technology and Physiology

CAHAG: COPD \& Astma Huisartsen Advies Groep (COPD \& Asthma GP Advisory Group)

SKL: Dutch Paediatric Repiratory Society

Education for Health, Warwick, UK

National Respiratory Training Center, VA, USA

NHG: Nederlands Huisartsen Genootschap (Dutch Society of GPs)

NVALT: Nederlandse Vereniging van Artsen voor Longziekten en Tuberculose (Dutch Thoracic Society)

NVLA: Nederlandse Vereniging Longfunctie Analisten

(Dutch Society of Respiratory Technicians)

PCRS-UK: Primary Care Respiratory Society UK

SSMG: Société Scientifique de Médecine Générale

WONCA: World Organization of Family Doctors

\section{Individuals}

Shawn Aaron, MD, Ottawa, ON, Canada

Peymané Adab, MB ChB, MPH, FFPH, FHKCCM, MD, Birmingham, UK

Sunil Kumar Agarwal MD, MPH, PhD, Raleigh-Durham, NC, USA

Ashutosh N. Aggarwal, MD, Chandigarh, India

Omar A. Al-Rawas, MD, Muscat, Sultanate of Oman

Santiago C. Arce, MD, Buenos Aires, Argentina

Bert G.M. Arets, MD, PhD, Utrecht, The Netherlands

Björn Bake, MD, PhD, Gothenburg, Sweden

Lutz Beckert, MB BS, MD, MRCP, FRACP, Christchurch, New Zealand

Helmi Ben Saad, MD, PhD, Sousse, Tunisia

Norbert Berend, Sydney, NSW, Australia

Erik Berglund, MD, PhD, Gothenburg, Sweden

Francesco Blasi, MD, Milan, Italy

Piotr W. Boros, MD, PhD, Warsaw, Poland

Otto Brändli, MD, Wald, Switzerland

Pierre-Olivier Bridevaux, MD, MSc, Geneva, Switzerland

Peter J. Briffa, BAppSc, GradDipNatRes, GradCertRespSc, MHltSc, CRFS, Camperdown, NSW, Australia

Ben Brockway, MD, Dunedin, New Zealand

Michael Brown, CRFS, Brisbane, QLD, Australia

Martyn Bucknall, London, UK

Johan Buffels, MD, PhD, Leuven, Belgium

Felip Burgos Rincón, RPFT, RN, Barcelona, Spain

Debbie Burton, BSc Hons, PhD, Rockhampton, QLD, Australia

Joyce Canterbury, RPFT, MEd., MHA, Denver, CO, USA

Richard Casaburi, PhD, MD, Torrance, CA, USA

Mario Cazzola, MD, Rome, Italy

Stefano Centanni, $\mathrm{MD}, \mathrm{PhD}$, Milan, Italy

Isa Cerveri, MD, Pavia, Italy

John Concato, MD, MPH, New Haven, CT, USA

Brendan G. Cooper, Consultant Clin Sci, Birmingham, UK

Ahmet S. Copur, NCHVAMC, North Chicago, IL, USA

Angelo G. Corsico, MD, PhD, Pavia, Italy 
John Cotes, MD, PhD, Durham, UK

Derek Cramer, BSc (Hons), MIScT, London, UK

Alan J. Crockett, PSM, MPH, PhD, FANZSRS, Adelaide, SA, Australia

Bruce H. Culver, MD, Seattle, WA, USA

Robert E. Dales, MD, MSc, Ottawa, ON, Canada

Wanchai Dejsomritrutai, MD, Bangkok, Thailand

P.N. Richard Dekhuijzen, MD, PhD, Nijmegen, The Netherlands

Eleonora Del Colle, BAppSci, CRFS, Box Hill, VIC, Australia

An De Sutter, MD, PhD, Ghent, Belgium

Helen Dunroy, MD, Dunedin, New Zealand

Derek Figurski, Laboratory Manager, Canberra, ACT, Australia

Monica J. Fletcher, Warwick, UK

Carlos A. Vaz Fragoso, MD, New Haven, CT, USA

Ashok Fulambarker, MD FCCP, Chicago, IL, USA

Kevin Gain, PhD, Perth, WA, Australia

Monika Gappa, MD, Wesel, Germany

Margaret W. Gerbase, MD, MSc, PhD, Geneva, Switzerland

John Gibson, BSc, MD, FRCP, Newcastle upon Tyne, UK

Thomas M. Gill, MD, New Haven, CT, USA

Kim Gooey, Respir. Scientist, Frankston, VIC, Australia

Christopher J. Gore, PhD, Canberra, ACT, Australia

Graham Hall, PhD, Perth, WA, Australia

James E. Hansen, MD, Torrance, CA, USA

Jon A. Hardie, MD, PhD, Bergen, Norway

John Henderson, MD, Bristol, UK

Eva Hnizdo, PhD, Morgantown, WV, USA

Guy Hollaert, MD, Dottignies, Belgium

Steven Holmes, MD, Shepton Mallet, UK

Frederic Hoppin, MD, Pawtucket, RI, USA

Birgitta Houltz, MD, PhD, Gothenburg, Sweden

Antoinette Houtkooper, BC, Den Helder, The Netherlands

Michael Hughes, DM, FRCP, London, UK

Georg Hülskamp, MD, Münster, Germany

Mark Hurwitz, Director Thoracic Medicine, Canberra, ACT, Australia

Robert Hyatt, MD, Rochester, MN, USA

Charles G Irvin, PhD, Burlington, VT, USA

David P. Johns, PhD, Hobart, TAS, Australia

Richard E. Kanner, MD, Salt Lake City, UT, USA

Hafsa Khaled, MD, Ksar Hellal, Tunisia

Jane Kirkby, PhD, London UK

Jana Kivastik, MD, PhD, Tartu, Estonia

Dieter Köhler, MD, Kloster Grafschaft, Germany

Nino Künzli, MD, PhD, Basel, Switzerland

S.M. Lam, MD, Hong Kong, SAR, China

Arnulf Langhammer, MD, PhD, Levanger, Norway

Le Thi Tuyet Lan, MD, Ho Chi Min City, Vietnam

Arthur C.W. Lau, MBBS, FHKAM (Medicine), FCCP,

Hong Kong, SAR, China

Mark L. Levy, MBChB, MRCGP, FRCGP, Kenton, UK

Carmen Lisboa, MD, Santiago, Chile

Sooky Lum, PhD, London, UK

Neil MacIntyre, MD, Durham, NC, USA

Peter T. Macklem, BA, MD, CM, FRCP, FRSC, Doct Hon Causa

Brussels \& Athens, Montreal, QC, Canada

Yves Martinet, MD, PhD, Nancy, France

Gail McAvay, PhD, New Haven, CT, USA

John McLachlan, MBChB (Cape Town), FCP(SA), FRACP,

CRFS, Hamilton, New Zealand
Astri Medbø, A Spes GP, PhD, Tromsø, Norway

Robert B. Mellins MD, New York, NY, USA

Peter J.F.M. Merkus, MD, PhD, Nijmegen, The Netherlands

Albert Miller, MD, New York, NY, USA

Carl Mottram, RRT, RPFT, Rochester, MN, USA

Pavlos Myrianthefs, MD, PhD, Kifissia, Greece

Jan Olofson, MD, PhD, R, Gothenburg, Sweden

Stanislaw Ostrowski MD, PhD, Lublin, Poland

Smita Pakhalé, MD, FRCPC, MSc (Epi \& Biostat), Ottawa, ON, Canada

Riccardo Pellegrino, MD, Cuneo, Italy

Carlos A.C. Pereira, MD, São Paulo, Brazil

Edward L. Petsonk, MD, Morgantown, WV, USA

Francesco Pistelli, MD, PhD, Pisa, Italy

K.V.V. Prasad, MD, Hyderabad, India

Jeff Pray, RRT, RPFT, Minneapolis, MN, USA

Jeffrey J. Pretto, Doc Hlth Sc, Newcastle, NSW, Australia

Neil B. Pride, MD, London, UK

Chengli Que, MD, PhD, Beijing, China

P. Sitarama Raju, MD, Hyderabad, India

Richard G. Roberts, MD, JD, Madison, WI, USA

David Robiony-Rogers, MBA, CRFS, Wellington, New Zealand

Nicolas Roche, MD, PhD, Paris, France

Leanne Rodwell, PhD, Brisbane, QLD, Australia

Margaret Rosenfeld, MD, MPH, Seattle, WA, USA

Bart Rottier, MD, Groningen, The Netherlands

Sonia Rouatbi, MD, Sousse, Tunisia

Richard E.K. Russell, MD, PhD, London, UK

Maureen Rutten-van Mölken, PhD, Maastricht, The Netherlands

Paul D. Scanlon, MD, Rochester, MN, USA

Tjard Schermer, MD, PhD, Nijmegen, The Netherlands

Thomas Similowski, MD, PhD, Paris, France

Iain R. Small, MD, Aberdeen, UK

Henk Stam, PhD, Rotterdam, The Netherlands

Sanja Stanojevic, PhD, London, UK

Irene Steenbruggen, RRT, RPFT, Zwolle, The Netherlands

Paul S. Stephenson, MA, FRCGP, Haverhill, UK

James Sullivan, BA, RPFT, New York, NY, USA

Madhavi Sunitha, MBBS, Hyderabad, India

Gerhard W. Sybrecht, MD, Homburg/Saar, Germany

Robin Taylor, MD, Dunedin, New Zealand

Frank Thien, MD, FRACP, Melbourne, VIC, Australia

Bruce R. Thompson, PhD, Melbourne, VIC, Australia

Waldemar Tomalak, MD, Rabka, Poland

Dominique Valeyre, MD, Bobigny, France

Jan Willem van den Berg, MD, Zwolle, The Netherlands

Jan Vandevoorde, MD, PhD, Brussels, Belgium

Peter H. Van Ness, PhD, MPH, New Haven, CT, USA

Onno van Schayck, PhD, Maastricht, The Netherlands

Chris van Weel, MD, PhD, Nijmegen, The Netherlands

Daphna Vilozni, PhD, Tel Aviv, Israel

William M. Vollmer, PhD, Portland, OR, USA

Karlman Wasserman, MD, PhD, Torrance, CA, USA

Stephen N. West, M App Sci, Sydney, NSW, Australia

Patrick White, Senior Lecturer, London, UK

Lisa Wilson, Respir Physiologist, Invercargill, New Zealand

Colin Wong, MD, Dunedin, New Zealand

E.F.M. Wouters, Maastricht, The Netherlands

Jan Zieliński, MD, PhD, Warsaw, Poland 


\section{Publications implicitly or explicitly critical of the use of the fixed $\mathrm{FEV}_{1} / \mathrm{FVC}$ ratio}

1 Quanjer PH, Tammeling GJ, Cotes JE, et al: Lung volumes and forced ventilatory flows. Eur Respir J 1993;6(suppl 16):5-40.

2 Falaschetti E, Laiho, Primatesta P, Purdon S: Prediction equations for normal and low lung function from the Health Survey for England. Eur Respir J 2004;23:456-463.

3 Stanojevic S, Wade A, Stocks J, et al: Reference ranges for spirometry across all ages: a new approach. Am J Respir Crit Care Med 2008; 177:253-260.

4 http://www.spirxpert.com/GOLD.html, downloading the software gives access to over 80 sets of prediction equations and over 50,000 data demonstrating how the GOLD criteria lead to underdiagnosis in young subjects and overdiagnosis in those over 50 years.

5 http://www.spirxpert.com/controversies/ controversy.html explains why the use of a fixed ratio is flawed.

6 Stanojevic S, Wade A, Stocks J: Reference values for lung function: past, present and future. Eur Respir J 2010;36:12-19.

7 Hardie JA, Buist AS, Vollmer WM, et al: Risk of over-diagnosis of COPD in asymptomatic elderly never-smokers. Eur Respir J 2002;20: 1117-1120.

8 Celli BR, Halbert RJ, Isonaka S, Schau B: Population impact of different definitions of airway obstruction. Eur Respir J 2003;22: 268-273.

9 Roberts SD, Farber MO, Knox KS, et al: $\mathrm{FEV}_{1} / \mathrm{FVC}$ ratio of $70 \%$ misclassifies patients with obstruction at the extremes of age. Chest 2006;130:200-206.

10 Aggarwal AN, Gupta D, Behera D, Jindal SK: Comparison of fixed percentage method and lower confidence limits for defining limits of normality for interpretation of spirometry. Respir Care 2006;51:737-743.

11 Miller MR, Steenbruggen I, Quanjer PH, et al: Defining the lower limit of normal for $\mathrm{FEV}_{1} / \mathrm{FVC}$. Letter to the Editor. Am J Resp Crit Care Med 2007;176:101-102.

12 Miller MR: What defines abnormal lung function? Letter to the Editor. Thorax 2007; 62:1107.

13 Petsonk EL, Hnizdo E, Attfield M: Definition of COPD GOLD stage I. Letter to the Editor. Thorax 2007;62:1107-1108.

14 Medbø A, Melbye H: Lung function testing in the elderly - can we still use the $\mathrm{FEV}_{1} / \mathrm{FVC}$ $<70 \%$ as a criterion for COPD? Respir Med 2007;101:1097-1105.

15 Shirtcliffe P, Weatherall M, Marsh S, et al: COPD prevalence in a random population survey: a matter of definition. Eur Respir J 2007:30:232-239.
16 Vollmer WM, Gíslason B, Burney P, et al: Comparison of spirometry criteria for the diagnosis of COPD: results from the BOLD study. Eur Respir J 2009;34:588-597.

17 Miller MR, Pedersen OF, Pellegrino R, Brusasco V: Debating the definition of airflow obstruction: time to move on? Editorial. Eur Respir J 2009;34:527-528.

18 Swanney MP, Ruppel G, Enright PL, et al: Using the lower limit of normal for the $\mathrm{FEV}_{1} /$ FVC ratio reduces the misclassification of airway obstruction. Thorax 2008;63:10461051.

19 Olofson J, Bake B, Tengelin MN, Houltz B COPD 'diagnosis' based on spirometric reference equations. Clin Respir J 2008;2:214219.

20 Ko FW, Woo J, Tam W, Lai CK, Ngai J, Kwok T, Hui DS: Prevalence and risk factors of airflow obstruction in an elderly Chinese population. Eur Respir J 2008;32:1472-1478.

21 Lau AC, Ip MS, Lai CK, Choo KL, Tang KS Yam LY, Chan-Yeung M: Variability of the prevalence of undiagnosed airflow obstruction in smokers using different diagnostic criteria. Chest 2008;133:42-48.

22 Schermer TR, Smeele IJ, Thoonen BP, et al: Current clinical guideline definitions for airflow obstruction leads to substantial overdiagnosis of COPD in primary care. Eur Resp J 2008;32:945-952.

23 Levy ML, Quanjer PH, Booker R, Cooper BG, Holmes S, Small I: Diagnostic spirometry in primary care: proposed standards for general practice compliant with American Thoracic Society and European Respiratory Society recommendations. Prim Care Resp J 2009;18:130-147.

24 Schermer TRJ, Quanjer PH: COPD screening in primary care: who is sick? Prim Care Respir J 2007;16:49-53.

25 Pérez-Padilla R, Hallal PC, Vázquez-García JC, et al: Impact of bronchodilator use on the prevalence of COPD in population-based samples. COPD: J Chron Obstruct Pulm Dis 2007;4:113-120.

26 Vaz Fragoso CA, Concato J, McAvay G, et al: The ratio of forced expiratory volume in 1-second to forced vital capacity as a basis for establishing chronic obstructive pulmonary disease. Am J Respir Crit Care Med 2010;181: 446-451.

27 Miller MR, Quanjer PH, Swanney MP, Ruppel G, Enright PL: Interpreting lung function data using 80 percent of predicted and 5 fixed thresholds misclassifies over $20 \%$ of patients. Published online before print June 3, 2010 doi: $10.1378 /$ chest.10-0189 chest.10-0189.
28 Enright PL: GOLD stage I is not a COPD risk factor. Letter to the Editor. Thorax 2007;62: 1107.

29 Miller MR, Pincock AC: Predicted values: how should we use them? Thorax 1988;43: 265-267.

30 ATS Statement: Lung function testing: selection of reference values and interpretative strategies. Am Rev Respir Dis 1991;144: 1202-1218.

31 Pellegrino R, Viegi G, Brusasco V, et al: Interpretative strategies for lung function tests. Series 'ATS/ERS Task Force: Standardisation of Lung Function Testing'. Eur Respir J 2005; 26:948-968.

32 Townsend MC: Conflicting definitions of airways obstruction: drawing the line between normal and abnormal. Editorial. Chest 2007;131:335-336.

33 Kerstjens HAM: The GOLD classification has not advanced understanding of COPD. Pro/Con Editorial. Am J Respir Crit Care Med 2004;170:212-213.

34 Enright P, Quanjer P: Don't diagnose mild COPD without confirming airway obstruction after an inhaled bronchodilator. Editorial. COPD 2007;4:89-90.

35 Culver $\mathrm{BH}$ : Interpretation of spirometry: we can do better than the GOLD standard. Editorial. Respir Care 2006;51:719-721.

36 Wilt T, Niewoehner DE, Kim CB, et al: Use of spirometry for case finding, diagnosis, and management of Chronic Obstructive Pulmonary Disease (COPD). Evidence Report/Technology Assessment No. 121 (Prepared by the Minnesota Evidence-based Practice Center under Contract No. 29002-0009.) AHRQ Publication No. 05-E0172. Rockville, MD. Agency for Healthcare Research and Quality, 2005.

37 Hnizdo E, Glindmeyer HW, Petsonk EL, et al: Case definitions for chronic obstructive pulmonary disease. COPD 2006;3:1-6.

38 Hansen JE, Sun XG, Wasserman K: Spirometric criteria for airway obstruction: use percentage of $\mathrm{FEV}_{1} / \mathrm{FVC}$ ratio below the fifth percentile, not $<70 \%$. Chest 2007;131:349355 .

39 Cerveri I, Corsico AG, Accordini S, et al: Underestimation of airflow obstruction among young adults using $\mathrm{FEV}_{1} / \mathrm{FVC}<70 \%$ as a fixed cut-off: a longitudinal evaluation of clinical and functional outcomes. Thorax 2008;63:1040-1045.

40 Cerveri I, Corsico AG, Accordini S, et al: What defines airflow obstruction in asthma? Eur Respir J 2009;34:568-573. 